Concurso

\section{Barrio Cívico}

\section{Concepción, Chile}

FECHA 2000

NÚMERO DE PROYECTOS RECIBIDOS SIN DATOS

DIRECCIÓN DEL CONCURSO FERNANDO MOSCOSO

JURADO IVANIA GOLES (PRESIDENTA), SERGIO BAERISWYL (REPRESENTANTE DEL

alCALDE), BERNARDo SUAZO (JEFE PRocrama RIBERA NORTE), CARLOS GONZÁLEZ

(REPRESENTANTE SEREMI VIVIENDA), FLAVIO VALASSINA (REPRESENTANTE DEL COLEGIO

DE ARQUITECTOS), JOSE LUIS LARROUCAU (SECRETARIO REGIONAL M.O.P.), SYLVIA

PIROTTE (ARQUITECTA DEL NIVEL CENTRAL DIRECCION DE ARQUITECTURA), JOSÉ

PICA (REPRESENTANTE DIRECTORA NACIONAL DE ARQUITECTURA), UIS IZQUIERDO

(PEPRESETTANTE DE LOS CONCURSAMTES), TERMANDO BASIDO (RERESENTANTE

MINISTERIO DE OO.PP.)

PESUITADOS PRIMER UUGAR. SMILAN RADIC, EDUABDO CASTILLO, EDUARDO SERPELL

SEGUNDO LUGAR: TEODORO FERNÄNDEZ, SEBASTIÄN HERNÁNDEZ, DANILO MARTIC, LUIS

ANTONIO MOMARES, MARTIN LABBEE, SANTIAGO ARIAS, EDUARDO VILHES.

TERCER LUGAR: RAMÓN ÓRDENES, CRISTIÁN CASTILLO, GENARO FERNÄNDEZ, ALFREDO

LIZANA, JOHN RATHKAMP, ERNESTO MUNOZZ

PRIMERA MENCIÓN HONROSA: CRISTIÁN PRADO, RAÚL ESPINOZA, JENS MONTT, JAVIER

CASTRO, CIRO NEIRA, MARCELA ROMAGNOU

MANDANTE DIRECCIÓN DE ARQUITECTURA MOP. VIII REGIONN
Cada obra tiene su historia, sus dificultades e inconvenientes. El nuevo Barrio Cívico plantea revitalizar el sector de la antigua Estación de Ferrocarriles dándole por uso la sede del Gobierno Regional. Una secuencia de cuatro pares de pabellones de oficinas -actualmente sólo un par ha sido construido- terminarán, finalmente, de consolidar integramente la idea original.

\section{PRIMER PREMIO}

Smiljan Radic Arquitecto, Pontificia Universidad Católica de Chile

Eduardo Castillo Profesor, Escuela de Arquitectura, Pontificia Universidad Católica de Chile y Universidad de Talca

Ricardo Serpell Profesor, Escuela de Arquitectura, Universidad Nacional Andrés Bello
Each work has its history, difficulties and problems. The new civic district aims to revitalize the area around the old railroad station, using it as the headquarters of the regional government. A series of four pairs of office blocks -only one of which has been built- will ultimately be the comprehensive realization of the original idea.

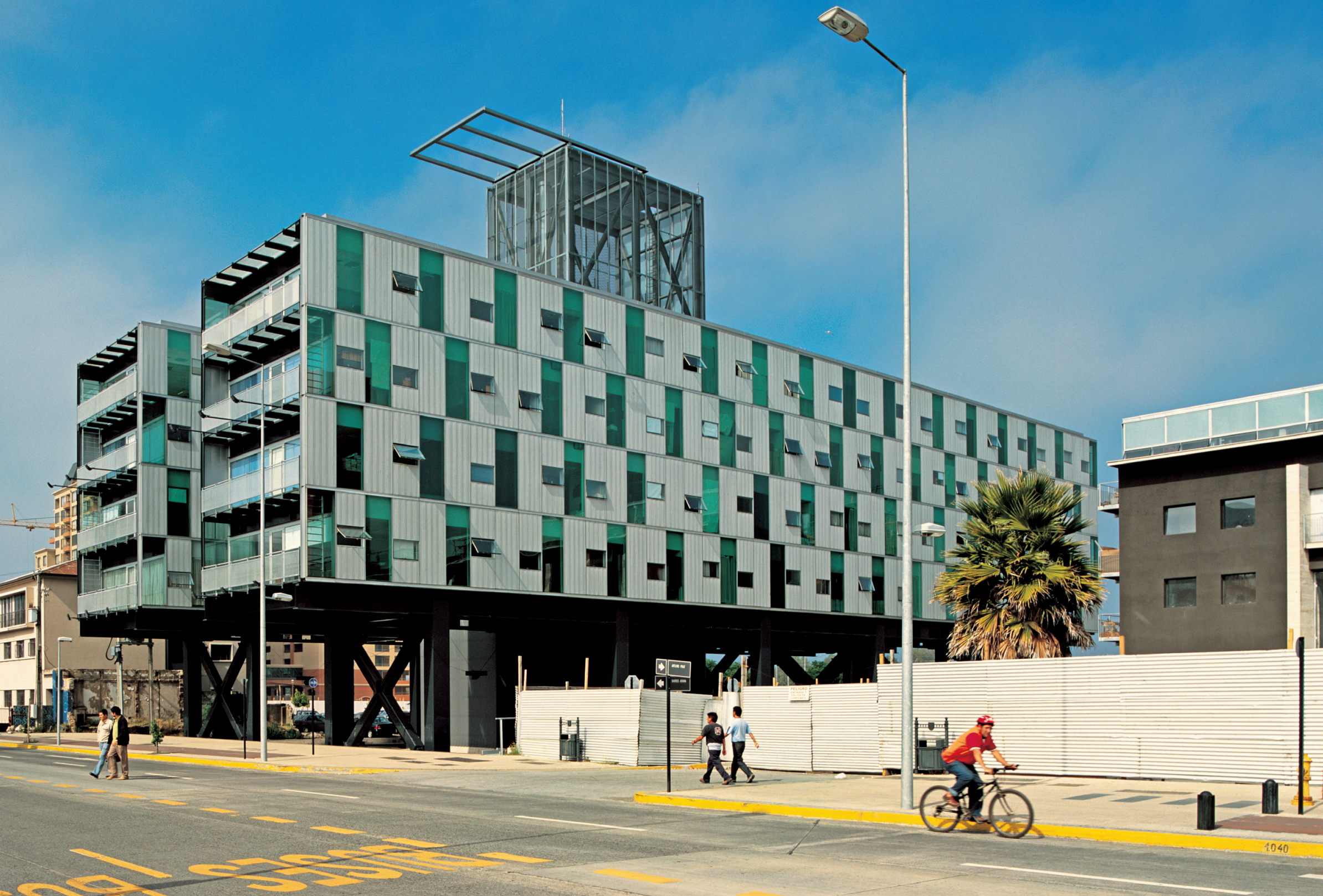



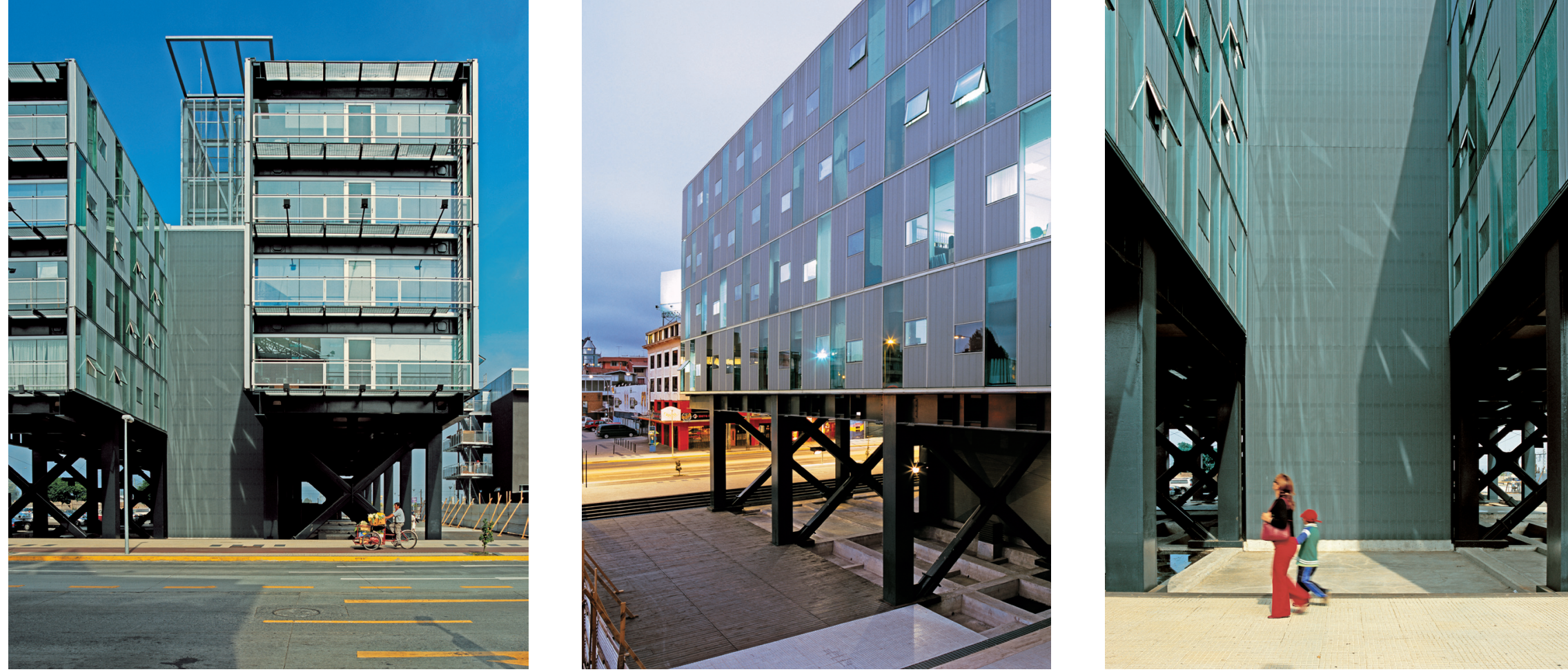

Nuestro proyecto para el Barrio Cívico de Concepción resultó ganador el año 2000 de un concurso nacional de arquitectura convocado por la Dirección de Arquitectura del Ministerio de Obras Públicas. Concebido para ser construido por etapas, hoy se han completado parcialmente dos de ellas: el reacondicionamiento de la Estación de Ferrocarriles, convertida en sede del Gobierno Regional y la construcción de las nuevas oficinas del M.O.P. De estas dos etapas, como siempre, se han dejado de edificar sus espacios públicos: una plaza de durmientes de tren, una laguna con juncos y un cine abierto. El proyecto ocupa un sitio intermedio entre la ciudad antigua nacida después del terremoto de 1939 y la nueva, por construirse sobre los sitios de la ribera norte del río Bío-Bío, ocupados hasta hace poco por las líneas ferroviarias. A ambos lados de la antigua Estación de Ferrocarriles se propusieron cuatro pares de edificios, en dirección río-ciudad. Estos pabellones convierten a la antigua estación en una pieza única y destacada, acentuando la frontalidad de su arquitectura de corte fascista.

En el interior de los nuevos pabellones de oficinas existe la sensación de estar en un único edificio unitario, a pesar de que el estrecho espacio dejado entre los pabellones, permite ver pasar la lluvia entre ellos.

Un nuevo par de módulos se construirá en el año 2008, manteniéndose su forma, pero cambiando la materialidad y la estructura de acero por una de hormigón armado en bruto. Así, el conjunto aparecerá matizado por los vaivenes del mercado y por las capacidades de sus proyectistas. ARQ

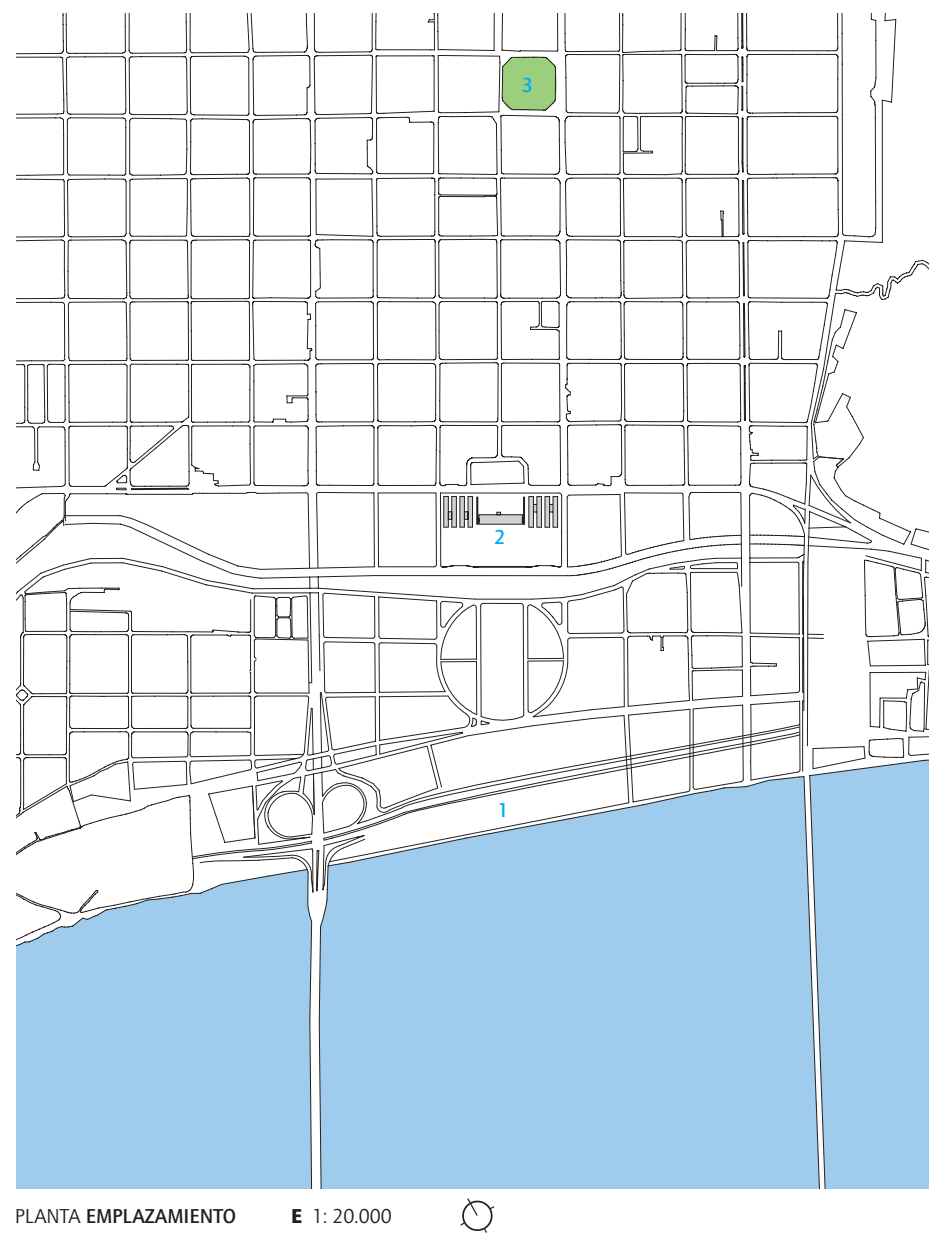

1 Rio Bío Bío

2 Barrio Cívico

3 Plaza de Armas 


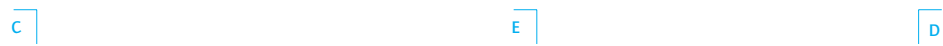

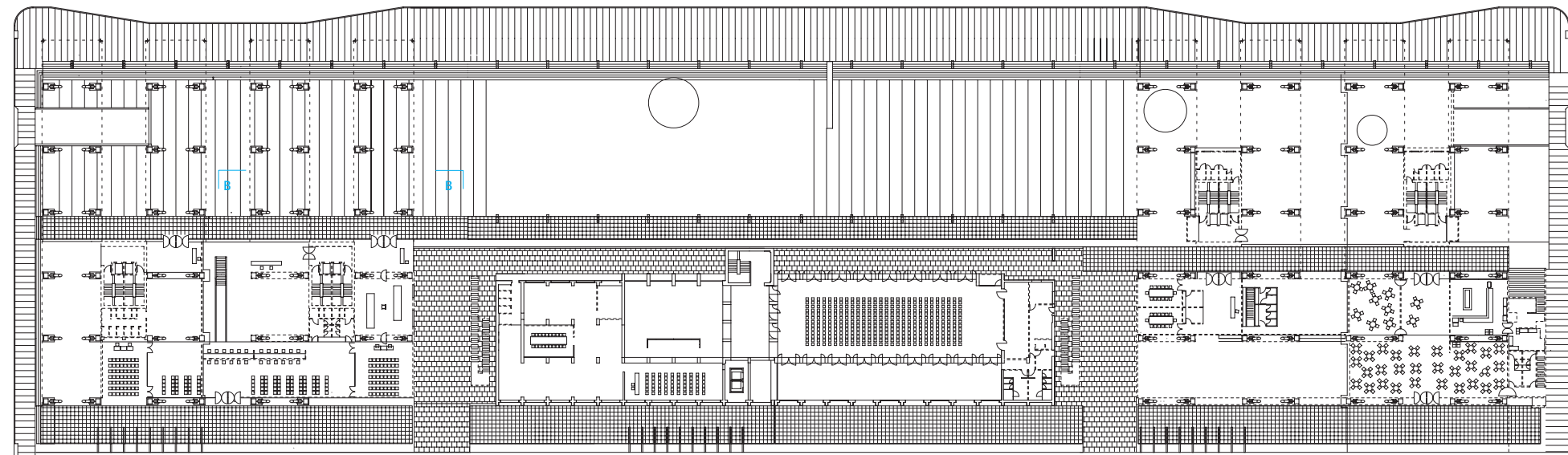

PLANTA PRIMER NIVEL E 1:1250 $\quad \bigcirc \quad c$

L

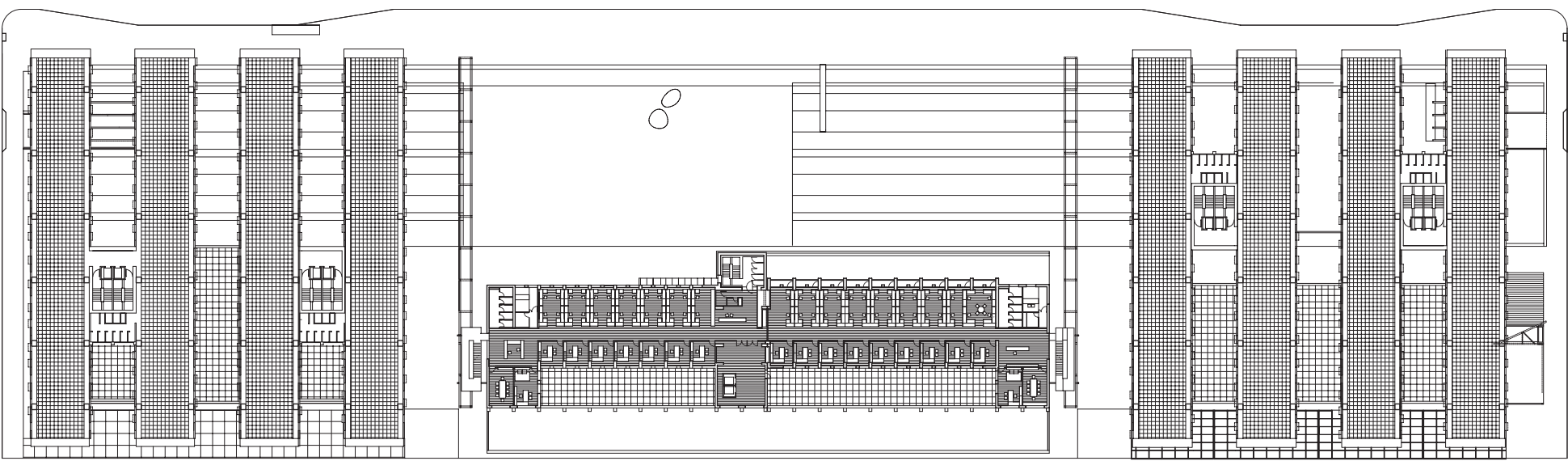

PLANTA TERCER NIVEL

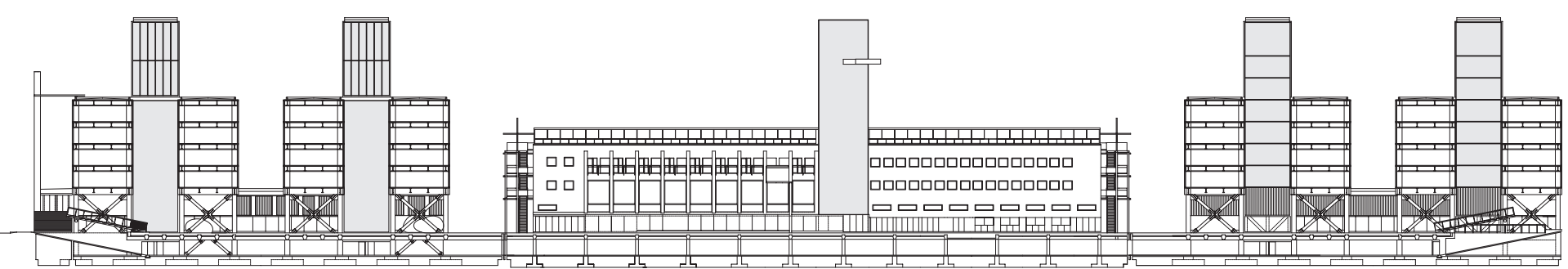

CORTE LONGITUDINAL AA 

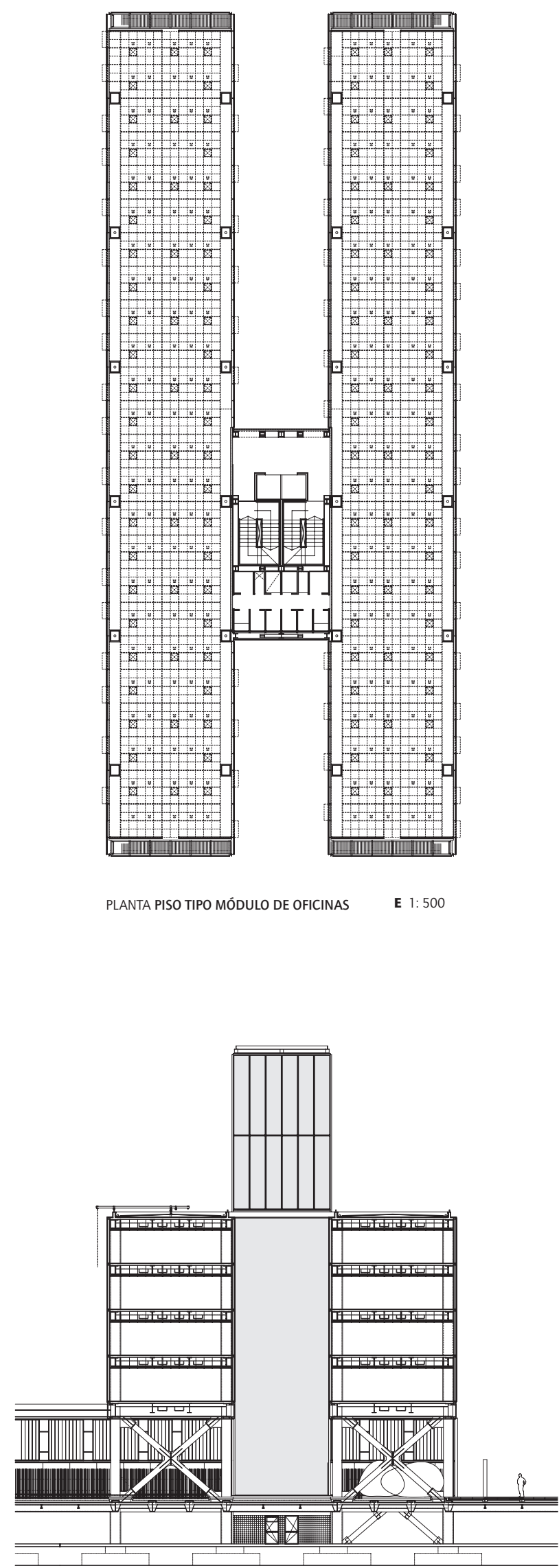

CORTE LONGITUDINAL BB 


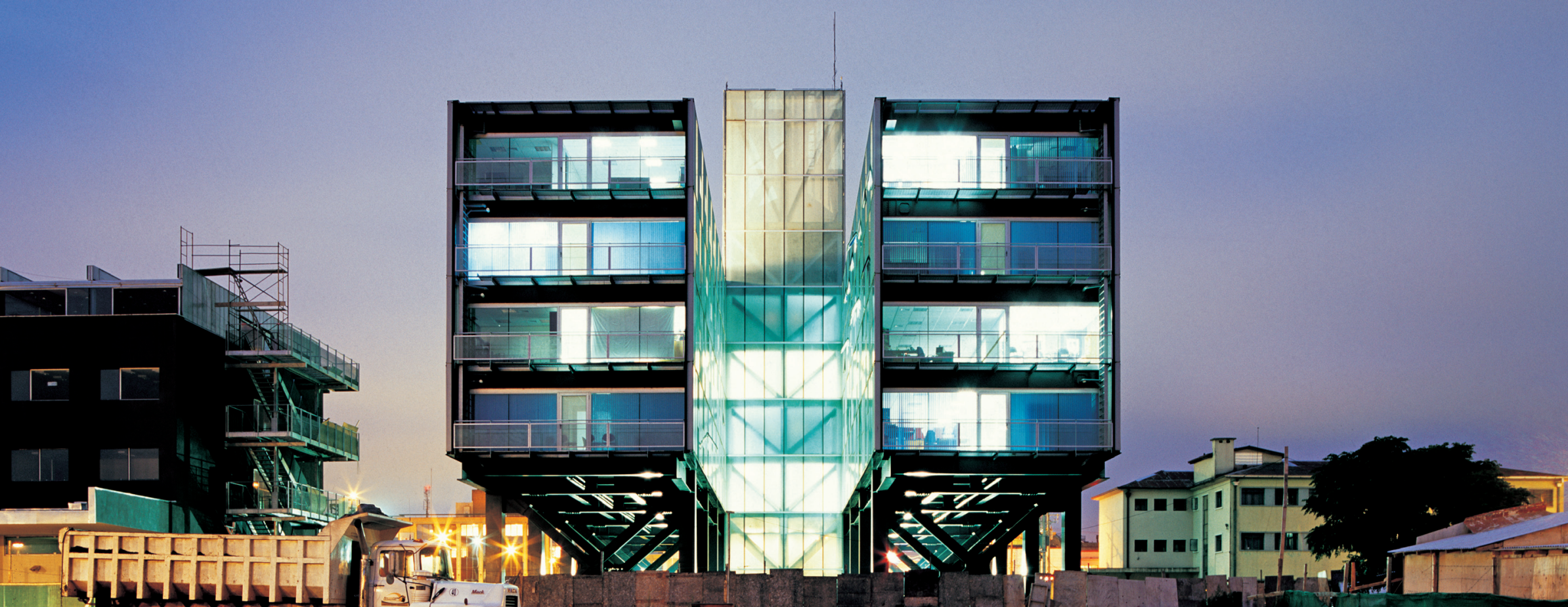

-

() (ㅇ) (영
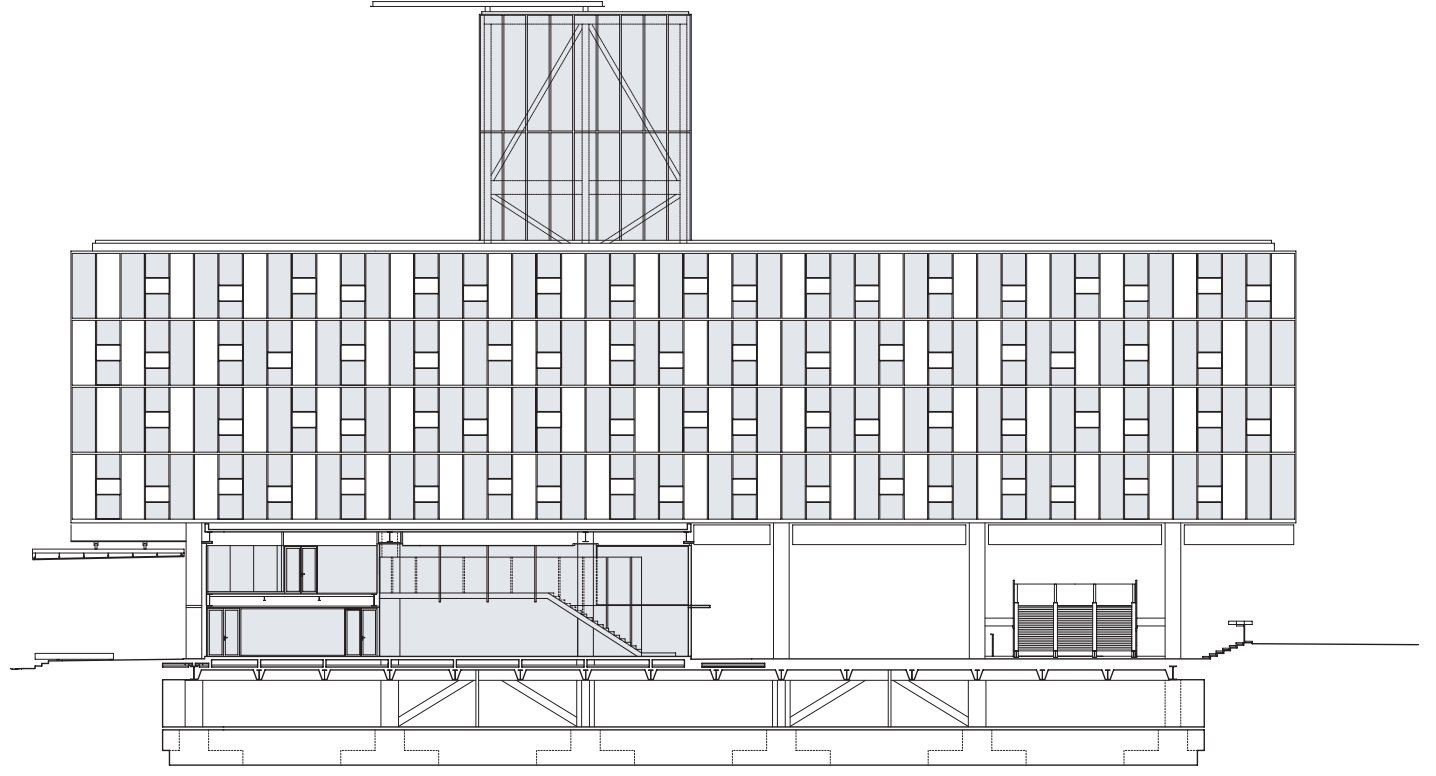

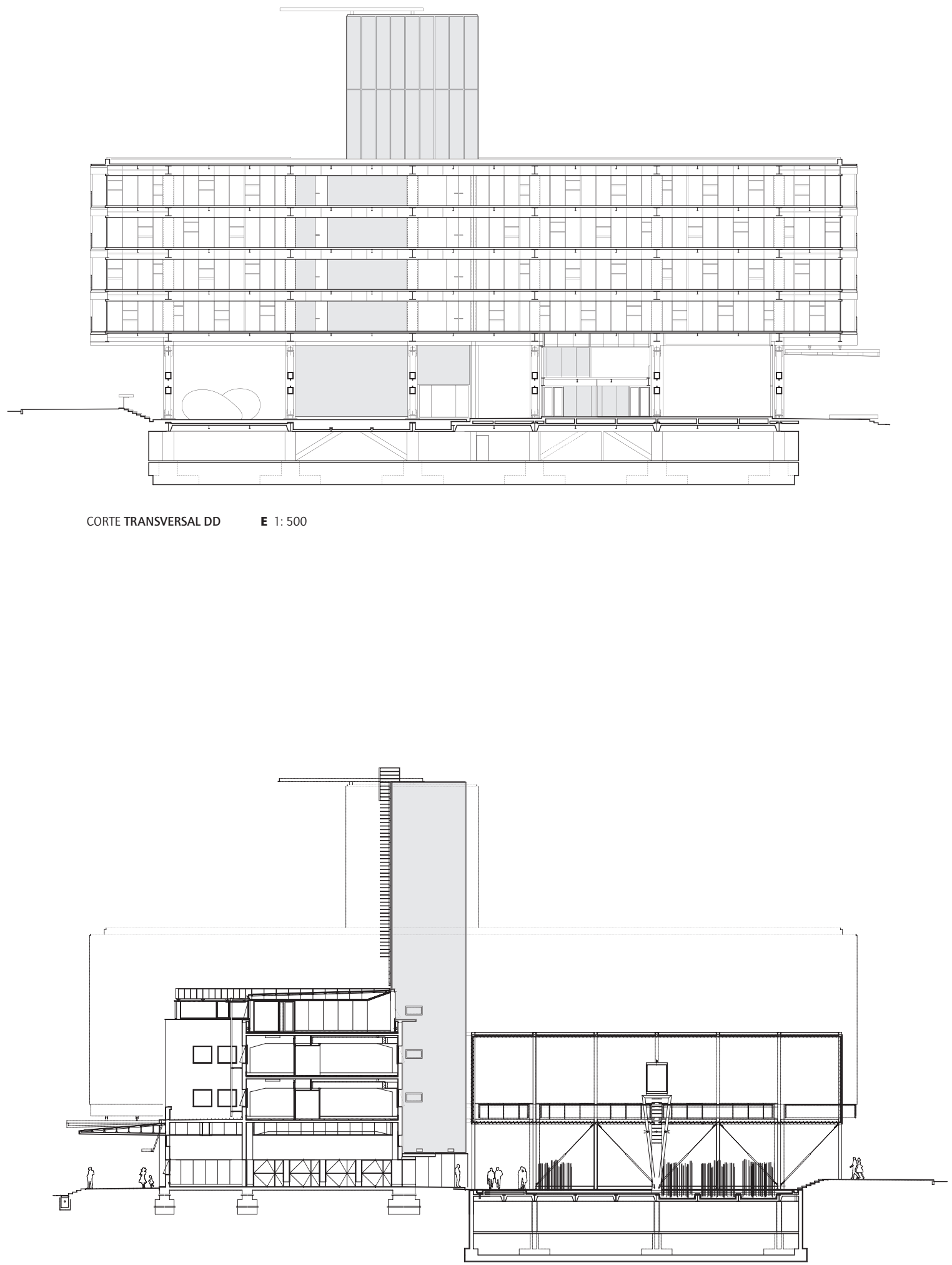

CORTE TRANSVERSAL EE 
